\title{
Random Access Compressed Sensing in Underwater Sensor Networks
}

\author{
Fatemeh Fazel \\ Northeastern University \\ Boston, MA 02115 \\ Email: ffazel@ece.neu.edu
}

\author{
Maryam Fazel \\ University of Washington \\ Seattle, WA 98195 \\ Email:mfazel@u.washington.edu
}

\author{
Milica Stojanovic \\ Northeastern University \\ Boston, MA 02115 \\ Email: millitsa@ece.neu.edu
}

\begin{abstract}
In this paper, we propose a power-efficient underwater sensor network scheme employing compressed sensing and random channel access. The proposed scheme is suitable for applications where a large number of sensor nodes are deployed uniformly over a certain area to measure a physical phenomenon. The underlying assumption is that most physical phenomena have sparse representations in the frequency domain. The network is assumed to have a Fusion Center (FC) that collects the observations of sensor nodes and reconstructs the measured field based on the obtained measurements. The proposed method is completely decentralized, i.e., sensor nodes act independently without the need for coordination with each other or with the FC. During each frame, a Bernoulli random generator at each node determines whether the node participates in sampling or stays inactive during that sampling period. If selected, it measures the physical quantity of interest, e.g. temperature. A second random generator with a uniform distribution then picks a (random) delay for the node to send its data to the FC. The proposed network scheme, referred to as Random Access Compressed Sensing (RACS), results in a simple power-efficient design, for: a) it eliminates the need for duplexing, which requires coordination from the FC; b) there is no need for acknowledgment packets and retransmissions in case packets collide; and moreover, c) it is efficient in terms of the communication resources used (only a small fraction of nodes sample and transmit in each sampling period).
\end{abstract}

\section{INTRODUCTION}

Sensor networks consist of a large number of sensor nodes that are deployed over a region of interest to observe the physical environment. Each sensor node communicates its observation of the field to a central node, referred to as the Fusion Center (FC) and the FC retrieves the information about the physical field. In this paper, we are interested in the case where the field of interest is sparse in some domain, noting that most natural phenomena are compressible (sparse) in an appropriate basis. The theory of compressed sensing establishes that under certain conditions on a signal, exact signal recovery is possible with a small number of random measurements [1],[2]. The application of compressed sensing in sensor networks has been studied in a number of references. Reference [3] introduces compressed cooperative spatial mapping using mobile networks and studies the minimal collective sensing needed to build an accurate map. Authors in [4],[5] and [6] use phase-coherent transmission of randomly-weighted data from sensor nodes to the FC, using a dedicated multipleaccess channel. Using this method, distributed projections of the sensor data into an appropriate basis is formed at the FC. Note that in this approach sensors need to be fully synchronized. In [7] the sensors are tracking the location of an audio source, transmitting their readings to an FC. In this setting, the signals appearing at each sensor are jointly sparse. The authors show that a very small number of measurements can achieve the signal detection goal. In reference [8] authors consider an on-off random multiple access channel where users communicate simultaneously, each with a certain probability, and the receiver must detect which users transmit. The authors transform the problem to an equivalent compressed sensing problem and use sparsity detection algorithms for finding the capacity bounds of the on-off random multiple access channel.

In this work, we consider a large underwater sensor network that observes a physical phenomenon for geographical and environmental monitoring purposes. We assume the physical phenomenon to be studied is compressible (sparse) in the frequency domain. Our proposed method, based on compressed sensing and random access, results in an efficient sampling and simple transmission scheme. Individual sensor nodes are not required to perform any processing, while most of the processing will be done at the FC. A typical sensor network scheme consists of (i) a sampling procedure, during which sensor nodes perform the measurement; followed by (ii) a channel access method, in order to transmit the measurements to the FC; and finally (iii) a recovery process, which is performed at the FC using sparsity based reconstruction algorithms. In the sampling procedure, we employ principles of compressed sensing to reduce the number of measurements required, moreover, in the channel access phase we propose a simple random access protocol. Random channel access can lead to packet losses due to collisions. Thus the fusion center obtains an incomplete set of measurements, due to (a) random sensing in the sampling phase, and (b) random losses due to the random access protocol. In order to reconstruct the complete field from an incomplete set of measurements at the FC, we use compressive sensing techniques. Note that our proposed method is completely distributed, requiring no coordination neither among sensor nodes nor among sensors and the FC.

The paper is organized as follows: In Section II we introduce our system model. In Section III, we propose both centralized and distributed sampling using compressed sensing. Section IV 
discusses the use of a simple random multiple access for transmission of data to the FC. In Section V, we employ compressive sensing techniques to recover the data from an incomplete subset of measurements. In Section VI, we offer a design approach that achieves a desired recovery probability at the FC. Finally, we provide concluding remarks in Section VII.

Notation: Throughout this paper, we use $\mathbb{R}$ and $\mathbb{C}$ to denote the set of real numbers and complex numbers, respectively. We let $\ell_{p}$ denote the $p$-norm of a vector $x=\left[x_{1}, \ldots, x_{N}\right]^{T}$ defined by $\|x\|_{\ell_{p}}=\left(\sum_{i=1}^{N}\left|x_{i}\right|^{p}\right)^{1 / p}$.

\section{SySTEM MODEL}

Let $f(x, t)$ denote the physical process of interest that the sensor network intends to measure, such as temperature, pressure, current, etc. We set up a linear network consisting of $N$ sensors that are uniformly distributed on a line. Note that the results of this paper can be extended to 2-dimensional (area) and 3-dimensional (volume) networks as well. The sensors are separated by a distance $d$ and conduct measurements every $T$ seconds as shown in Figure 1. We assume that the network has a Fusion Center (FC) with the task of collecting the measurements and reconstructing the field of interest. In order to determine the appropriate value for $T$, we look at the correlation properties of the underlying physical process. We assume that $f(x, t)$ is a wide-sense stationary signal and its autocorrelation can be approximated as

$$
\begin{aligned}
R_{f f}(\Delta x, \Delta t) & =E\{f(x+\Delta x, t+\Delta t) f(x, t)\} \\
& \approx R_{1}(\Delta t) R_{2}(\Delta x)
\end{aligned}
$$

Let us define the coherence time $T_{c o h}$ of $f(x, t)$ as the time difference over which the process almost de-correlates in time, i.e. $R_{1}\left(T_{\text {coh }}\right) / R_{1}(0)=X \%$, where e.g. $X=10$. A conventional design parameter for a network measuring $f(x, t)$ is then to set $T=T_{\text {coh }}$ i.e. to obtain new measurements every $T_{\text {coh }}$ seconds. Let $L$ denote the number of bits per packet of data. Also, assume that each sensor has a fixed bandwidth $B$ to communicate with the FC. Therefore, each data packet takes $T_{p}=\frac{L}{B}$ seconds to be transmitted. The propagation delay of each sensor's packet depends on the distance between the sensor node and the FC. Let $D_{i}$ denote the distance of node $i$ from the FC, where $i \in\{1, \ldots, N\}$. The propagation delay corresponding to node $i$ 's packet is given by $\tau_{i}=\frac{D_{i}}{c_{0}}$, where $c_{0}=1500$ meters/sec is the nominal speed of sound. Throughout the rest of the paper, we assume that the sensor nodes are placed on the sea floor while the FC is located on the surface of a body of water with depth $D$, where $D \gg N d / 2$; therefore, we can assume that $D_{1} \approx D_{2} \approx \ldots \approx D_{N}=D$.

Let us define the coverage area of a network as the total area covered by the sensor network. In our network model the coverage area is given by $N d$.

\section{A. Conventional (Benchmark) Network}

In a conventional sensor network all $N$ nodes, separated by distances $d$, conduct measurements every $T_{c o h}$ seconds,

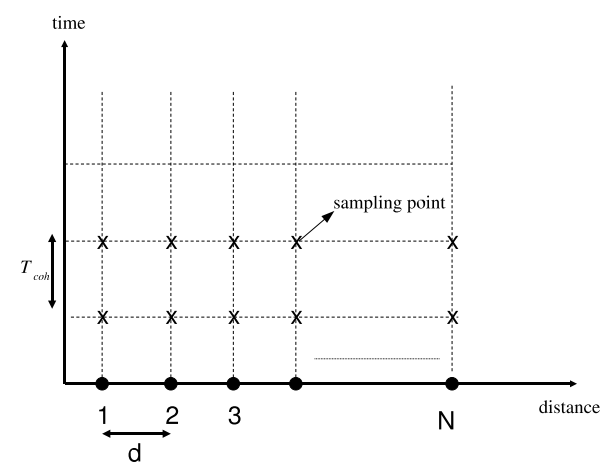

Fig. 1. Linear network model and a conventional design approach where all nodes perform sampling every $T=T_{\text {coh }}$ seconds

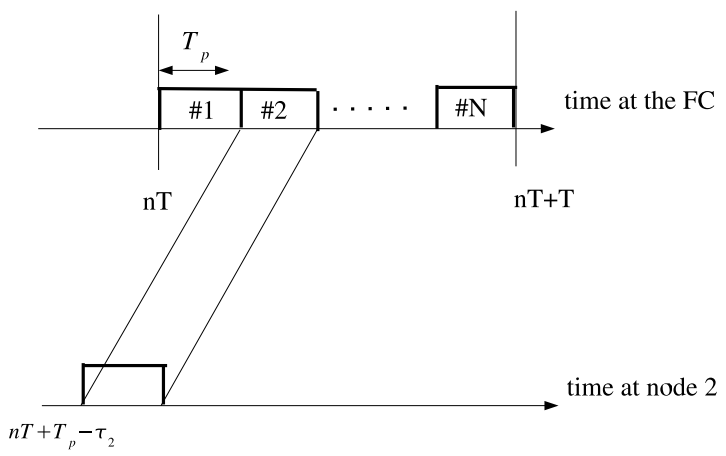

Fig. 2. The scheduling required at each node in TDMA

and transmit the measurements to the FC using a multipleaccess scheme. In the rest of this paper, we assume the conventional network employs the standard TDMA scheme to transmit data packets to the FC. This requires nodes to schedule their transmissions such that at the FC, each node's packet is received back-to-back to the previous node's packet. Figure 2 demonstrates the scheduling procedure.

The total number of nodes that a conventional network can support is given by

$$
N \leq \frac{T_{c o h}}{T_{p}}
$$

where $T_{c o h}$ is the property of the underlying physical process (new information is needed every $T_{c o h}$ seconds). Denoting $N_{\text {conv }}=T_{\text {coh }} / T_{p}$, the coverage area of a conventional network is thus limited to $A=N_{\text {conv }} d^{2}=T_{c o h} d^{2} / T_{p}$.

The process of data gathering consists of two phases: sensing and communication. The sensing phase can be (i) deterministic (conventional case), meaning that all sensors sample the physical phenomenon, or (ii) random (compressed), 
meaning that only a random subset of sensor nodes participate in sampling. In the communication phase, nodes that have taken part in sensing, communicate their measurements to the FC using a multiple access scheme. Multiple-access techniques are generally divided into two categories: i) deterministic access methods, e.g. TDMA, FDMA, CDMA; and ii) random access methods, e.g. Aloha, CSMA and CSMA/CD. Typically, deterministic access methods are used in networks where users have a steady flow of information, whereas if users have bursty information, random access methods are preferred. In deterministic methods, if the transmitter has no data to send the channel remains idle, in such situations random access provides an efficient mechanism for accessing the channel.

\section{SAMPLING PROCEDURE}

At each frame $n$, we denote node $i$ 's measurement by $u_{i}(n)=f\left(x_{i}, t_{n}\right)$, where $i \in\{1, \ldots, N\}$. The complete map of the process is denoted by $\mathbf{u}(n)=\left[u_{1}(n) \ldots u_{N}(n)\right]^{T} \in$ $\mathbb{R}^{N}$ which contains the measured quantities at all sensor locations. Let $\mathbf{U}(n)$ denote the Fourier transform of $\mathbf{u}(n)$. Now $\mathbf{u}(n)=\Psi \mathbf{U}(n)$ where $\Psi \in \mathbb{C}^{N \times N}$ is the inverse discrete Fourier transform matrix. Most natural phenomena have compressible (sparse) representation in the frequency domain, hence we assume that $\mathbf{U}(n)$ is sparse. Note that a sparse signal is a signal that can be represented by a small number of non-zero coefficients, compared to the dimension of the signal. When a signal is sparse, based on the theory of compressed sensing, it can be recovered from a small subset of random measurements [1], [2]. At frame $n$, a subset of sensors is selected to conduct measurements. Note that the data vector $\mathbf{u}(n)$ is in spatial domain. Let $\Phi$ denote the sensing basis, i.e. the domain in which we perform the sensing. By randomly selecting sensors, we perform the sensing directly in the spatial domain, hence $\Phi=\mathbf{I}_{N \times N}$. Let $y(n) \in \mathbb{C}^{M \times 1}$ denote the observations of a random subset of $M$ sensors, and $z(n)$ represent the noise due to sensing and communication between the sensors and the FC. The received data vector at FC can be expressed as

$$
y(n)=R(n) \mathbf{u}(n)+z(n)
$$

where $R(n) \in \mathbb{R}^{M \times N}$ contain $M$ uniformly selected rows of $\Phi$ i.e. each of its $M$ rows contains a single 1 at the position of a selected sensor while all the other elements are zero. Furthermore, $\Psi$ represents the domain in which $\mathbf{u}(n)$ has a sparse representation. Therefore, Eq. (3) can be re-written in terms of the sparse vector $\mathbf{U}(n)$ as follows:

$$
y(n)=R(n) \Psi \mathbf{U}(n)+z(n)
$$

In the reconstruction procedure, one tries to recover the vector $\mathbf{U}(n)$ as accurately as possible and reconstruct the measured field $\mathbf{u}(n)=\Psi \mathbf{U}(n)$.

The coherence between the sensing basis $\Phi$ and the representation basis $\Psi$ is defined by [1]:

$$
\mu(\Phi, \Psi)=\sqrt{N} \max _{1 \leq k, j \leq N}\left|\left\langle\Phi_{k}, \Psi_{j}\right\rangle\right|
$$

where it can be shown that $\mu(\Phi, \Psi) \in[1, \sqrt{N}]$. Note that in the case of Eq. (3), where $\Phi=\mathbf{I}_{N \times N}$ and $\Psi$ is the inverse Fourier transform matrix, the coherence is derived as $\mu(\Phi, \Psi)=1$. In other words, the $(\Phi, \Psi)$ pair is maximally incoherent. Reference [1] states that for a signal with sparsity $S$, if we select $M$ measurements uniformly at random where $M \geq C \mu^{2} S \log N$ for some positive constant $C$, solving

$$
\min \|\mathbf{U}(n)\|_{\ell_{1}} \quad \text { subject to } \quad y(n)=R(n) \Psi \mathbf{U}(n)
$$

recovers the signal with overwhelming probability. A variety of algorithms for solving this optimization problem as well as other recovery methods have been studied [9].

\section{A. Centralized Sensing}

In the centralized scheme, a central scheduler at the FC determines a random subset of $M$ sensors to perform the sampling. This method requires the FC to broadcast the selected set of nodes to all the sensors. The selected nodes then sample $f(x, t)$ and send their measurements back to FC using a multiple-access technique. Because the FC broadcasts the selected sensors, all nodes learn which sensors transmit and in what order. Therefore, the network can simply use deterministic access (TDMA) with $M$ slots. All transmitting nodes organize their transmissions such that they are received at the FC in the requested order. One frame duration thus consists of the round trip broadcast time followed by $M$ packets of data, as shown in Figure 3, therefore $T=2 \tau+M T_{p}$ where $\tau=\frac{D}{c_{0}}$ denotes the propagation delay in the network. Moreover, the network needs updated data every $T_{c o h}$ seconds, therefore the frame duration $T$ must be less than or equal to the coherence time, in other words $M T_{p}+2 \tau \leq T_{\text {coh }}$.

\section{B. Distributed Sensing}

Centralized selection requires scheduling among sensors by downlink transmission from the FC. In order to eliminate the need for downlink transmissions at each frame, we propose a simple scheme to decentralize the process of selecting a random subset of nodes. We simply equip each sensor node with a Bernoulli random generator, generating independent identically distributed Bernoulli random variables, $X_{1}, \ldots, X_{N}$, where for all $i \in\{1, \ldots, N\}$,

$$
X_{i}=\left\{\begin{array}{l}
1 \\
0 \quad \text { with probability } p \\
\text { with probability } 1-p
\end{array}\right.
$$

The total number of sensors selected for transmission, $M$, is now given as

$$
M=\sum_{i=1}^{N} X_{i}
$$

which follows a Binomial distribution with parameters $N$ and $p$, i.e., $M \sim B(N, p)$. Now the probability density function of $M$ is given as

$$
P_{M}(m)=\operatorname{prob}(M=m)=\left(\begin{array}{l}
N \\
m
\end{array}\right) p^{m}(1-p)^{N-m}
$$

In this case, deterministic access can no longer be used, however, that is of no concern because as we will see in section IV, 


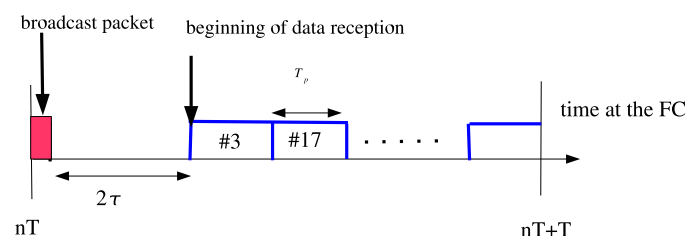

Fig. 3. The frame structure when using centralized sensing and TDMA

we can couple random channel access with distributed random sensing.

\section{Channel Access Procedure}

As a simple and efficient multiple access scheme, we investigate the use of random access as a means to accommodate random transmissions. The common random access schemes include Aloha, slotted Aloha and CSMA/CD. Note that these protocols rely on ACK (acknowledgment packets) to ensure that data is transmitted successfully. However, in an RACS network, once the FC has obtained a sufficient number of measurements, it can successfully reconstruct the data and there is no need to ensure that all packets are received successfully. The key idea here is to let FC simply discard the colliding packets. This approach is motivated by the compressed sensing theory and the fact that the FC does not care which specific sensors are selected as long as (i) the selected subset is chosen uniformly at random and (ii) there are sufficiently many collision-free packets received at the FC to allow for the reconstruction of the field. Therefore, in RACS scheme once a collision is detected FC simply discards the colliding packets and reconstructs the data using the rest of the packets. Note that a collision is said to have occurred if packets from different sensors overlap in time. Figure 4 depicts the random access scheme.

Assume that each sensor picks a random transmission delay $\theta_{i}$ uniformly distributed in $\left[0, T-T_{p}\right]$. Let us assume that $m$ sensors are selected for transmission. The average number of packet arrivals at the FC per unit of time is given by $\lambda=$ $\frac{m}{T-T_{p}}$. In order to determine the probability of collision, let us denote the number of packet arrivals in $[0, t]$ by the random variable $\mathcal{N}(t)$. Note that $\mathcal{N}(t)$ has a Poisson distribution with parameter $\lambda t[10]$, given by

$$
\operatorname{prob}(\mathcal{N}(t)=\mathcal{K})=\frac{(\lambda t)^{\mathcal{K}}}{\mathcal{K} !} e^{-\lambda t}
$$

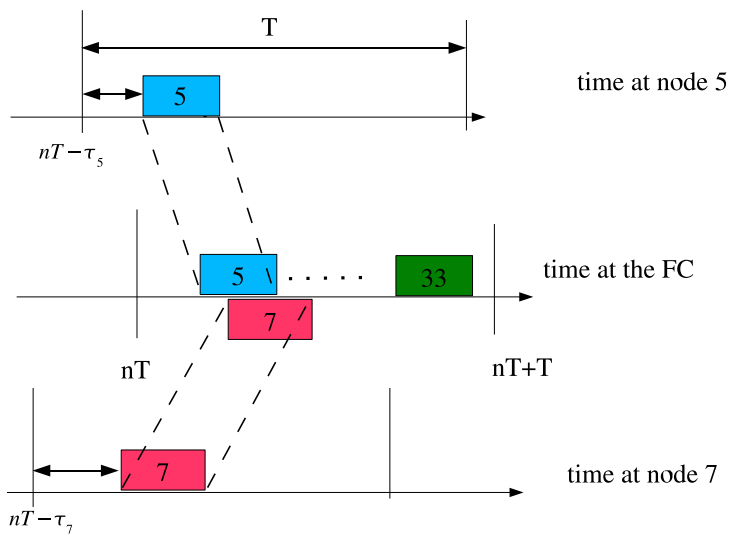

Fig. 4. Random channel access; packets from nodes 5 and 7 collided at the FC

where $\lambda t$ is the average number of packets in $[0, t]$. If a user $i$ transmits with a delay of $\theta_{i}$, its packet will occupy $\left[\theta_{i}, \theta_{i}+\right.$ $T_{p}$. A collision happens if another user transmits at any time $\left[\theta_{i}-T_{p}, \theta_{i}+T_{p}\right]$. Probability of no-collision is thus given by $e^{-2 \lambda T_{p}}$. Therefore, probability of collision in a network with uniformly-distributed transmission time in $\left[0, T-T_{p}\right]$ is given by

$$
p_{c o l}=1-e^{-2 \frac{m T_{p}}{T-T_{p}}}
$$

The proposed RACS protocol is summarized below:

i) at the beginning of frame $n$, sensor node $i$ determines whether it participates in sensing (with probability $p$ ) or stays inactive (with probability $1-p$ ) during that frame

ii) if node $i$ is not selected for sampling it stays inactive until the next frame $n+1$

iii) if node $i$ is selected for sampling, it measures the physical quantity of interest and encodes it into a packet of size $L$. The sensor's location is also appended to the packet.

iv) node $i$ runs a uniform random generator which determines the delay $\theta_{i}$, uniformly distributed in $\left[0, T-T_{p}\right]$, for the sensor's transmission of its packet

v) FC collects the packets received during $[n T, n T+T]$

vi) if a collision is detected, FC discards the colliding packets

vii) at the end of the frame, at time $n T+T$, FC uses the correctly received packets to reconstruct the data using $\ell_{1}$ minimization (or other sparsity based reconstruction methods). We assume packets that do not collide are correctly received.

\section{DATA RECOVERY}

Let $K$ denote the number of correctly received packets at the FC and $N_{s}=C S \log (N)$ denote the number of observations required to allow accurate reconstruction. In general determining $N_{s}$ analytically depends on the value of the constant $C$, a theoretical upper-bound for which is offered in [11]. 


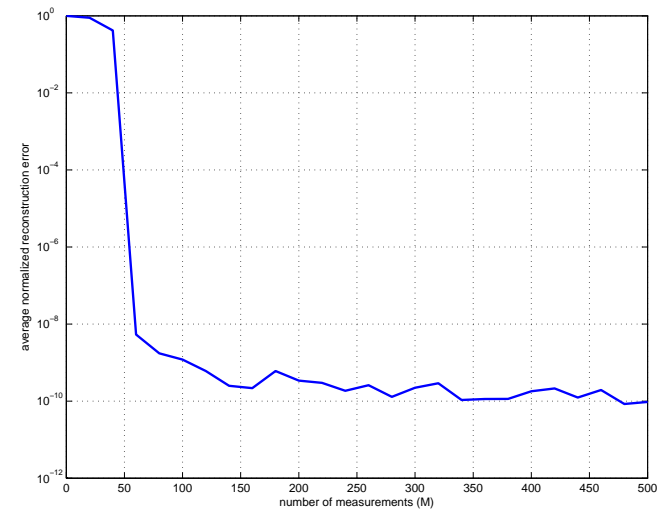

Fig. 5. The average normalized reconstruction error is plotted vs. the number of measurements $(M)$, for a signal of size $N=1000$ and with a sparsity of $S=10$. The desired number of measurements $N_{s}$ to obtain error-free reconstruction can be determined from the figure.

However, one can empirically determine $N_{s}$ as the number of measurements for which the reconstruction error is negligible. It turns out that the empirical value of $N_{s}$ is typically much smaller than the one obtained following the theoretical $C$. As an example, assume that we generate random sparse signals with a size $N=1000$ and a sparsity of $S=10$ and study the recovery of the signal for different number of measurements in a noise-free setting. Figure 5 shows the average reconstruction error vs. the number of measurements. As seen in the figure, for approximately $M \geq 70$ recovery is attained with an error below $10^{-8}$. Hence, a reasonable choice for $N_{s}$ is determined as $N_{s}=70$.

In the centralized sensing, ignoring packet losses due to channel fading, the number of received packets is the same as the number of transmitted packets $(K=M)$. Thus choosing $M=N_{s}=C S \log (N)$ provides a sufficient number of packets at the FC. In this case, the number of nodes $N$ that can be deployed in the network is determined as

$$
\left.\begin{array}{c}
M T_{p}+2 \frac{D}{c_{0}} \leq T_{\text {coh }} \\
M \geq C S \log (N)
\end{array}\right\} \Rightarrow \log (N) \leq \frac{T_{c o h}-2 \frac{D}{c_{0}}}{C S T_{p}}
$$

In comparison with the conventional scheme in Eq. (2), we have that $N \leq e^{\frac{1}{C S}\left(N_{c o n v}-2 \frac{D}{c_{0} T_{p}}\right)}$. Consequently, by using centralized random sensing jointly with TDMA as the channel access method, significantly more nodes can be deployed in the network than in the benchmark case. Since sensor nodes are separated by a fixed sensing distance $d$, by increasing the number of sensors we can extend the coverage area of the network. Moreover, total power consumption of the network is reduced by a factor of $\frac{N}{M}$ where $M=C S \log (N)$. Note that the coverage extension and power saving are achieved at the cost of additional downlink communication from the FC. In the distributed sensing case however, $K$ and $M$ are both random variables. The fact that $K$ is a random variable now has the following implication: There is no guarantee that $K$ will be larger than $N_{s}=C S \log (N)$ i.e. accurate reconstruction can not be guaranteed, however, by choosing $p$ carefully, reconstruction with a certain probability $P_{s}$ is possible.

Assume $m$ sensors are selected for sampling, resulting in $k$ correctly received packets at the $\mathrm{FC}$, where $k \leq m$. Therefore, to ensure that $N_{s}$ packets arrive at the FC collision-free, the probability of sensor selection $p$ has to be such that the number of selected sensors $m$ is greater than the desired number of observations $N_{s}$. This brings us to the question of how $p$ should be selected to enable reconstruction with a certain probability. We will propose a design approach in section VI, but first let us look at some examples.

\section{A. Numerical Examples}

We consider a linear network consisting of $N=1000$ equally spaced sensor nodes. Assume the physical quantity of interest is fully-sparse in the frequency domain with a sparsity of $S=10$. Furthermore, assume $T_{c}=120$ seconds, each sensor is given a transmission bandwidth of $B=5$ Kbps and each packet has a size of $L=1000$ bits. Figure 6 shows the number of collision-free received packets as a function of the sensing probability $p$. As seen in the figure, there is an interplay between the number of measurements and the number of collisions. While increasing $p$ results in a greater number of measurements $m$ and thus could improve the accuracy of reconstruction, however, it also increases the probability of collision and after a certain point may even decrease the number of collision-free packets received at the FC and affect negatively on the reconstruction quality. Figure 7(a) plots the average normalized reconstruction error as a function of $p$ for a randomly generated sparse data. As noted in the figure, accurate reconstruction is possible for a range of values of $p$. Figure 7(b) shows the corresponding average power consumption of the network as a function of $p$. In order to minimize the power consumption of the network while maintaining the quality of reconstruction (on the average), we choose the smallest value of $p$ for which accurate reconstruction is possible. In our simulations, we used CVX [12], a package for specifying and solving convex programs, to solve the $\ell_{1}$-minimization.

\section{Design APPROACH}

Let $P_{K}(k)=\operatorname{prob}\{K=k\}$. The overall probability distribution function for $K$ is given by

$$
\begin{aligned}
P_{K}(k) & =\sum_{m=k}^{N} \operatorname{prob}(K=k \mid M=m) \operatorname{prob}(M=m) \\
& =\quad \sum_{m=k}^{N}\left(\begin{array}{c}
m \\
k
\end{array}\right)\left(1-p_{c o l}\right)^{k} p_{c o l}^{m-k} P_{M}(m)
\end{aligned}
$$

where $P_{M}(m)$ is given by Eq. (8). The above expression does not appear tractable. We thus turn to finding an approximation for $P_{K}(k)$. We conjecture that $K$ is binomial like $M$, with the same $N$ but a probability $q<p$, i.e. $K \sim B(N, q)$. This is intuitively pleasing because the received packets are 


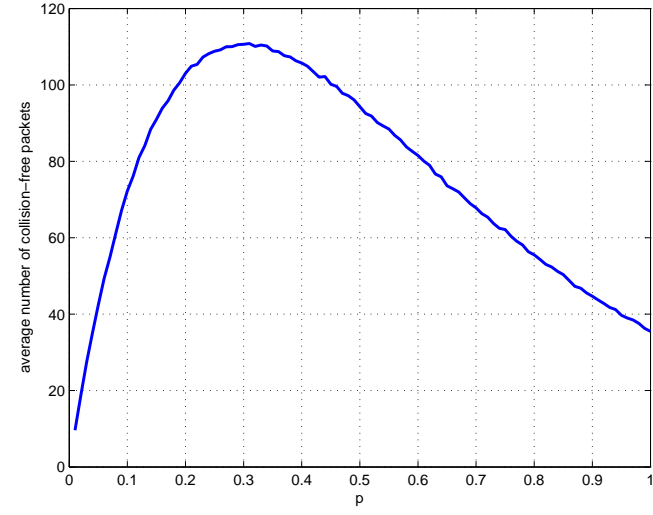

Fig. 6. Average number of collision-free received packets $\bar{K}$ vs. $p$; simulation parameters are $N=1000, T_{c o h}=120$ seconds and $T_{p}=0.2$ second.

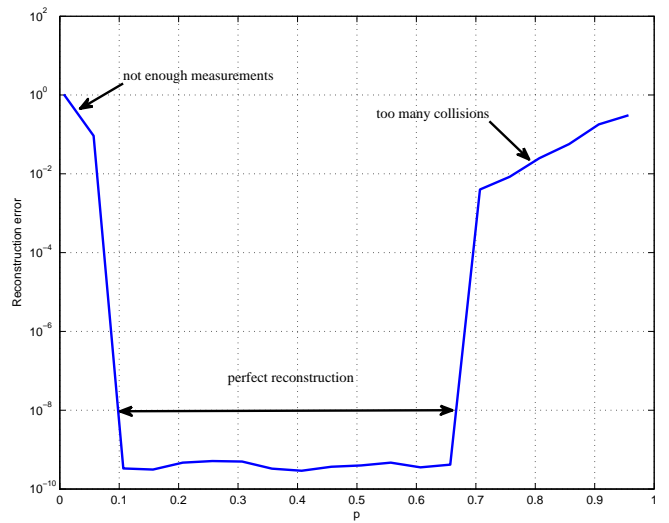

(a) reconstruction error

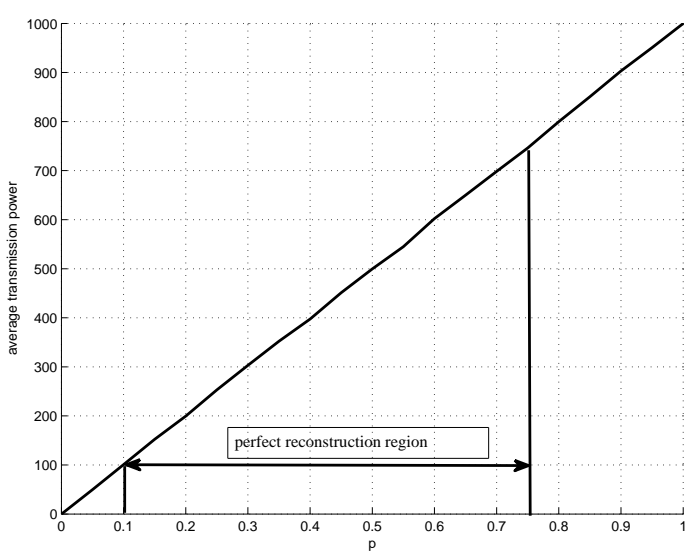

(b) average power consumption

Fig. 7. Average normalized reconstruction error vs. $p$ (a) and the corresponding power consumption (b); within the region where perfect reconstruction is possible we pick the smallest $p$ resulting in the least power consumption

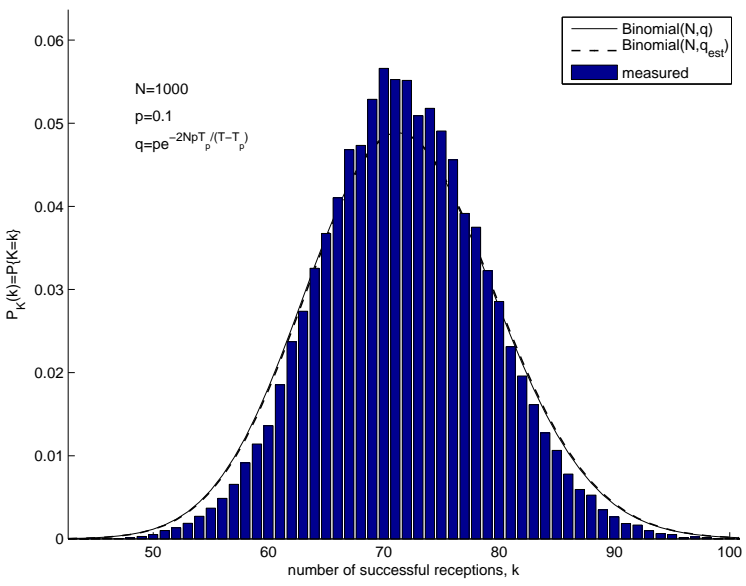

Fig. 8. Probability density function; Simulation parameters are $N_{\text {sim }}=$ 50000 simulation runs, $T_{p}=0.2 \mathrm{sec}$ and $T_{\text {coh }}=120 \mathrm{sec}$.

the same as transmitted packets minus random collisions. To empirically verify the conjecture, we simulate the process and count the number of successfully received packets. Figure 8 shows the probability density function of $K$ for an example set of system parameters. In this figure, the $P_{K}(k)$ obtained from measurements is compared with that of an estimated model $B\left(N, q_{e s t}\right)$ and a hypothesized model $B(N, q)$ where

$$
q_{\text {est }}=\frac{\frac{1}{N_{\text {sim }}} \sum_{i=1}^{N_{\text {sim }}} k(i)}{N}
$$

where $k(i)$ is the number of successfully received packets in the $i$-th simulation run and $N_{\text {sim }}$ is the total number of runs, and

$$
q=p e^{-2 \frac{N p T_{p}}{T-T_{p}}}
$$

We note that $q$ and $q_{\text {est }}$ are very close and the resulting binomial distributions closely match that of $K$, thus the $K$ process indeed seem to follow the binomial distribution and there is very good agreement between the histogram and $B(N, q)$. Figure 9 shows the complementary cumulative probability function $Q_{K}(k)$ from measurements, as well as for $B(N, q)$ and $B\left(N, q_{\text {est }}\right)$.

For a given $N$, let $N_{s}=C S \log (N)$ denote the number of packets needed for reconstruction. We define the probability of sufficient sensing as

$$
\operatorname{prob}\left\{K \geq N_{s}\right\}=Q_{K}\left(N_{s}\right)
$$

Let us define $P_{s}$ as the desired recovery probability, meaning we would like the recovery to happen at the FC with probability $P_{s}$. We need to determine $q_{s}$ such that

$$
Q_{K}\left(N_{s}\right) \geq P_{s} \quad \text { for } \quad q \geq q_{s}
$$

Now, let $q=p e^{-\alpha p}$, where $\alpha=2 \frac{N T_{p}}{T-T_{p}}$. The so-obtained value of $q_{s}$ is then used to determine the underlying $p$ and $\alpha$ required to maintain Eq. (12). There are multiple solutions to this equation. We want to identify the ones such that $T \leq$ $T_{c o h}$. Moreover, our design approach is to minimize the power 


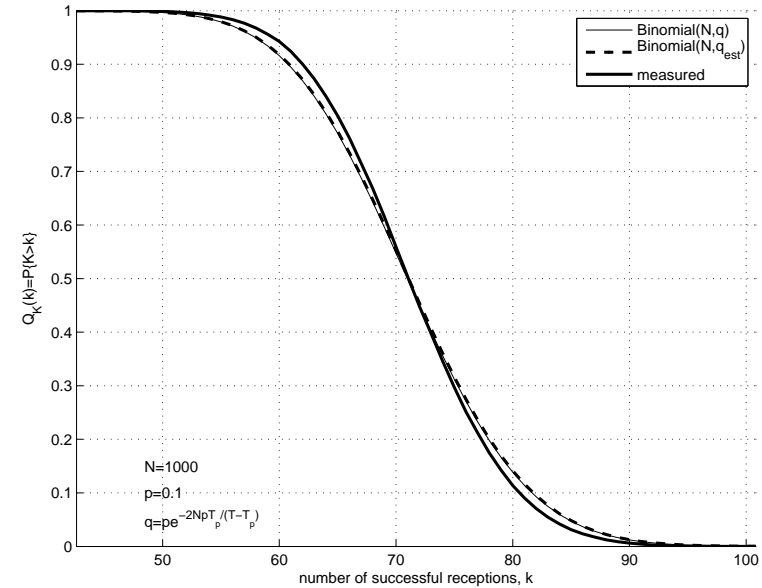

Fig. 9. Complementary cumulative function; Simulation parameters are $N_{\text {sim }}=50000$ simulation runs, $T_{p}=0.2 \mathrm{sec}$ and $T_{\text {coh }}=120 \mathrm{sec}$.

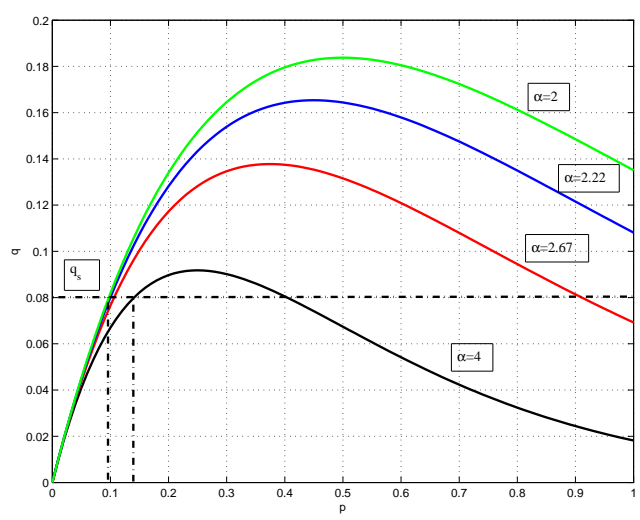

Fig. 10. $\mathrm{q}(\mathrm{p}, \alpha)$ vs. $\mathrm{p}$ for different values of $\alpha$; For a given $q_{s}$ a smaller $\alpha$ results in a smaller $p$.

consumption, hence we want the solution yielding the smallest $p$. Figure 10 shows plots of $q(p, \alpha)$ for different values of $\alpha$. As we see in this figure, for a given $q_{s}$, the curve with a smaller $\alpha$ yields a smaller solution for $p$. Hence, the smaller the $\alpha$ the smaller the probability $p$. The smallest $\alpha$ is determined using $T=T_{c o h}$ to be

$$
\alpha_{\min }=\frac{2 N T_{p}}{T_{c o h}-T_{p}} .
$$

Thus, the first step in the design approach is to solve for $q$ in Eq. (12). This can be done numerically for a given $N$. The second step is to find the underlying $p$ using the hypothesized model for $q$ given by $q=p e^{-p \alpha_{\text {min }}}$, where $\alpha_{\text {min }}$ is given by Eq. (13). In summary, we have a design approach that avails itself to a simplified model. Starting with a given number of sensors and a frame size $T \leq T_{\text {coh }}$, this approximate model is used to determine the sensing probability $p$ such that recovery happens with a desired probability $P_{s}$ at the FC.

\section{CONCLUSION}

In this paper, we proposed a simple power-efficient sensor network scheme, denoted as RACS, which employs random sensing and random channel access to deliver a subset of sensor measurements to the FC. Coupling random access with random sensing, we eliminated the need for duplexing. We then used compressed sensing techniques to recover the field from this random subset of measurements. Furthermore, given a desired recovery probability $P_{s}$, we provided a design methodology to determine the sensing probability $p$ such that the FC recovers the field with the desired probability. Under the assumption that most physical phenomena have compressible (sparse) representation in the frequency domain, we showed that the proposed RACS scheme is capable of recovering the measured field with a desired probability, using considerably less resources than a conventional network. Note that power is a scarce resource in an underwater sensor network due to the limited battery life of the nodes, therefore, saving power can extend the life-time of a network.

\section{REFERENCES}

[1] E. J. Candes and M. B. Wakin, "An introduction to compressive sampling," IEEE Signal Processing Magazin, pp. 21-30, March 2008.

[2] R. Baraniuk, "Compressive sensing," IEEE Signal Processing Magazin, pp. 118-121, July 2007.

[3] Y. Mostofi and P. Sen, "Compressive cooperative sensing and mapping in mobile networks," in Proceedings of the American Control Conference (ACC), St. Louis, Missouri, June 2009.

[4] W. Bajwa, J. Haupt, A. Sayeed, and R. Nowak, "Compressive wireless sensing," in 5th Int. Conf. Information Processing in Sensor Networks (IPSN'06), April 2006, pp. 134-142.

[5] W. Bajwa, A. Sayeed, and R. Nowak, "Matched source-channel communication for field estimation in wireless sensor networks," in 4th Int. Conf. Information Processing in Sensor Networks (IPSN'05), April 2005, pp. $332-339$.

[6] W. Bajwa, J. Haupt, A. Sayeed, and R. Nowak, "Joint sourcechannel communication for distributed estimation in sensor networks," IEEE Transactions on Information Theory, vol. 53, no. 10, pp. 3629-3653, October 2007

[7] A. Griffin and P. Tsakalides, "Compressed sensing of audio signals using multiple sensors."

[8] A. K. Fletcher, S. Rangan, and V. K. Goyal, "On-off random access channels: A compressed sensing framework," Submitted to IEEE Transactions on Information Theory.

[9] J. Tropp and S. Wright, "Computational methods for sparse solution of linear inverse problems," Proceedings of the IEEE, vol. 98, pp. 948-958, 2010.

[10] A. Leon-Garcia, Probability and Random Processes for Electrical Engineering, 2nd ed. Addison-Wesley, 1993.

[11]

[12] M. Grant and S. Boyd, "CVX: Matlab software for disciplined convex programming," http://cvxr.com/cvx/. 